\title{
Culture visuelle et pensée architecturale
}

Visual culture and Architectural Thought

\section{Stéphanie Dietre}

\section{(2) OpenEdition}

\section{Journals}

Édition électronique

URL : http://journals.openedition.org/crau/408

DOI : $10.4000 /$ crau. 408

ISSN : 2547-5746

Éditeur

Éditions du patrimoine

Édition imprimée

Date de publication : 1 décembre 2014

Pagination : 153-162

ISBN : 978-2-7577-0379-3

ISSN : 1296-4077

Référence électronique

Stéphanie Dietre, "Culture visuelle et pensée architecturale », Les Cahiers de la recherche architecturale et urbaine [En ligne], 30/31 | 2014, mis en ligne le 14 septembre 2017, consulté le 21 avril 2019. URL : http://journals.openedition.org/crau/408; DOI : 10.4000/crau.408 


\section{Culture visuelle et pensée architecturale}

A partir de notre travail de thèse en architecture, sous la direction de Catherine Maumi ${ }^{1}$, interrogeant I'architecture comme science du visible, nous souhaitons développer notre réflexion sur les moyens offerts à l'architecte pour transformer sa culture visuelle en pensée de l'architecture et en outils du projet.

\section{L'architecture est une science de la représentation}

Vitruve établit que l'architecture est la science de l'architecte: " architecti scientia». C'est une science car, dit-il, elle manifeste ce qui est signifié et ce qui signifie: "quod significatur et quod significat ». L'architecture est donc une science de la représentation et, précise Vitruve, une science de la représentation, grâce à la science du dessin: "graphidis scientia ».

Le travail engagé sur l'émergence du visible interrogée par les architectes, part de l'hypothèse du dessin comme système d'apprentissage de l'architecte. Cette question mène à proposer la «visibilité » que Konrad Fiedler énonce à la fin du XIXe siècle, à

1 Thèse soutenue au sein de l'école doctorale 454 "Sciences de l'homme, du politique et du territoire » de l'université de Grenoble et du laboratoire « Les métiers de l'histoire de l'architecture » de l'Ensa de Grenoble. 
partir de la peinture comme théorie de la connaissance, en tant que notion architecturale également. Grâce au contexte philosophique de la Sichtbarkeit ${ }^{2}$ - «visibilité »-, nous nous posons la question de la transformation de la culture visuelle de l'architecte vers une culture architecturale spécifique. Le travail de thèse considérant le « dessin de note » comme formulation d'une pensée non verbale, dans une forme courte de représentation, nous a amenée à réfléchir sur la représentation comme manifeste du regard instruit de l'architecte. "Pour voir il faut tout autre chose qu'un regard aigu ou attentif, il faut un regard instruit, un regard renouvelé, lui-même produit par une réflexion du " changement de terrain " sur l'exercice du voir ${ }^{3}$. » En tant que culture et pensée, le regard est ce qui est convoqué dans le savoir pour voir. C'est en acte et subi en même temps. La note est un choix, intelligible et/ou sensible. C'est la marque du plus important. L'architecte prend le parti de retenir ce qui l'étonne et ce qu'il cherche à voir, ce qu'il donne à voir et comment il le met en forme. La note est le système graphique que l'architecte met en place pour étudier l'existant et recueillir ses recherches. Lorsqu'il note en dessin, I'architecte cherche, observe tout en se laissant émouvoir et surprendre. II admet l'attitude heuristique lacanienne de l'attention flottante et progresse en conscience de son étonnement. L'architecte qui travaille à s'instruire selon son propre arbitre et ses propres moyens, peut renouveler son regard et par là même son savoir, en considérant ce qui l'étonne. Car ce qui anime l'homme à la recherche, cette "subite surprise de l'âme ${ }^{4}$ », reste "l'étonnement». « C'est en effet par l'étonnement, nous dit Aristote au
Livre A de la Métaphysique, que les humains, maintenant aussi bien qu'au début, commencent à philosopher $^{5}$. » La note est l'outil minimal qui s'adapte à la rapidité de l'avènement soudain d'une idée et d'un étonnement. Elle a cette double capacité de saisir et de réduire, de retenir graphiquement l'extraordinaire et ses objets admirables qui «meuvent les sens" parce qu'ils semblent rares. Le dessin devient intellection s'il manifeste de la Darstellung - impression - et de la Vorstellung - expression. II est la monstration et la transformation d'une culture visuelle en vision (culture et pensée) architecturale.

\section{La question du visible}

L'émergence du visible comme intérêt philosophique à la fin $d u$ XIX siècle, participe d'une interaction nouvelle entre art, science et technique ${ }^{6}$. À partir des avancées scientifiques de la Révolution industrielle sur le monde de la vision, les artistes engagent une exploitation industrielle du travail visuel. Ils posent scientifiquement la question du visible, reconnaissant sa spécificité comme outil et pensée. Dans le contexte de la transformation par l'industrialisation, des arts et des sociétés, Charles-Édouard Jeanneret, Paul Tournon et Jean-Charles Moreux se forment par le dessin et la note en dessin. Ils ont la même manière particulière d'utiliser le dessin alors que leurs méthodes graphiques restent très différentes. Pour l'exercice de leur formation puis de leur métier, ils progressent dans une réflexion sur les sciences visuelles et le dessin comme réunion des arts graphiques.
2. Konrad Fiedler, Aphorismes. Édition consultée, établie par Danièle Cohn pour la traduction de Sacha Zilberfarb, Paris, Images modernes, 2004.

3. Louis Althusser, Lire Le Capital, I, 1968. Édition consultée, Paris, Maspero, 1973, p. 18
4. René Descartes, Fuvres et lettres. Les passions de l'âme, seconde partie, 1649. Édition consultée, Paris, Gallimard, coll. "Bibliothèque de La Pléiade», 1952, p. 723-795.
5. Aristote, Ta meta ta physika, [982a. 10]. Édition consultée, Marie-Paule Duminil et Annick Jaulin pour la traduction française du grec ancien, Métaphysique, "Livre A. Des traités métaphysiques », "La sagesse est sa propre fin », [982a. 10], Paris, Flammarion, coll. «GF», 2008, p. 77.

6. Eugène Emmanuel Viollet-le-Duc, Histoire d'un dessinateur. Comment on apprend à dessiner, Paris, Hetzel et Cie, 1879. Édition consultée, Paris, Berger-Levrault, 19789. 


\section{Paul Tournon}

Paul Tournon s'instruit en analysant théoriquement, par la note en dessin, des grands édifices publics manifestes de l'histoire de l'architecture classique. Il apprend à maîtriser la science classique du plan. L'architecte acquiert, grâce à la note en dessin, une habilité dans le plan. La note devient une aide à la compréhension et à la conception parce qu'elle engage la réversibilité analyse/projet. Avec les méthodes graphiques académiques, Tournon cherche à montrer directement sa pensée des choses observées. Le travail d'abstraction de la note en dessin, offre à l'architecte le moyen d'utiliser les matériaux et les techniques de la modernité, selon un regard classique du plan. Ceci Cela lui permet de parfaire le projet de l'édifice et de son insertion urbaine. Tournon pense l'architecture comme machine graphique et visuelle, particulièrement en interaction avec des artistes et des artisans graphiques.

(Fig. 1)

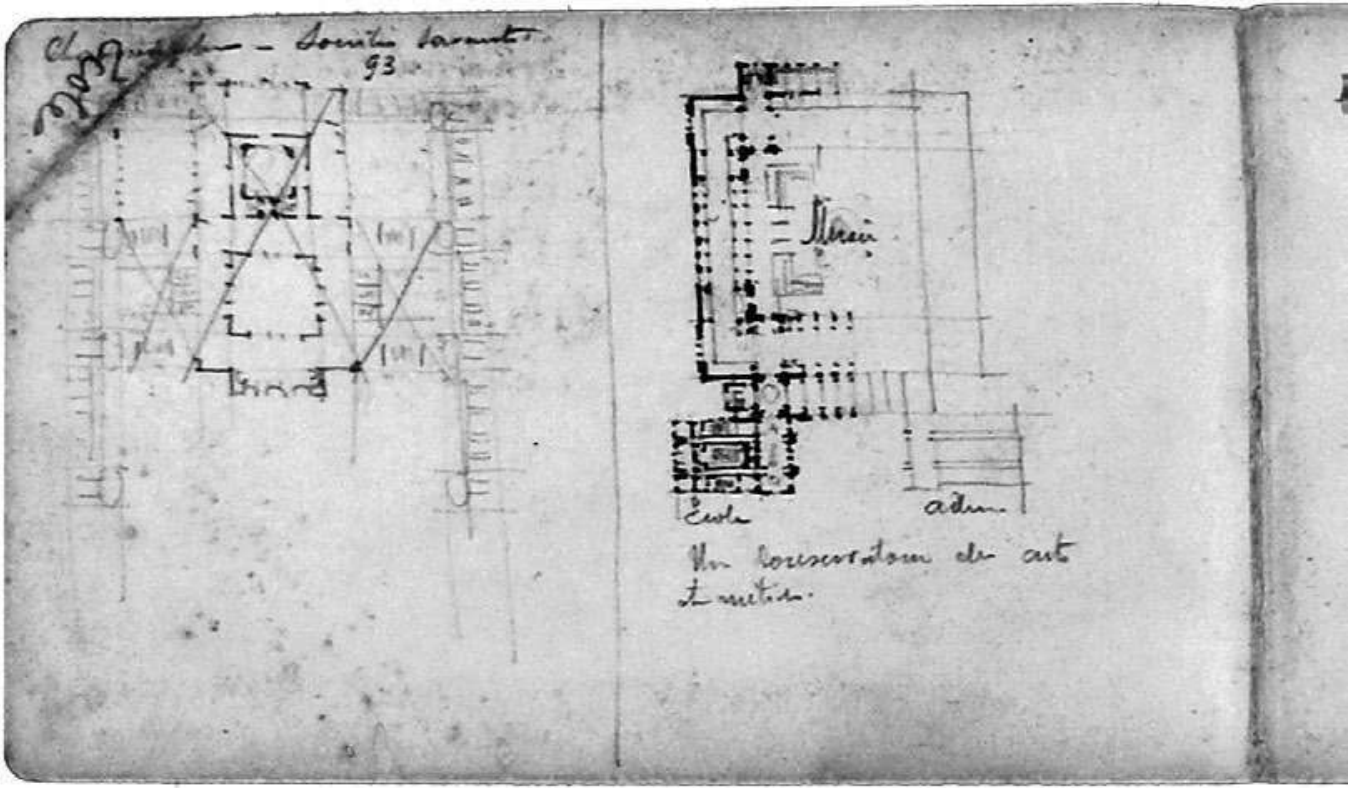

[fig. 1]. Paul Tournon, « La science du plan ", dessin à la mine de plomb sur papier quadrillé, carnet $10 \times 15 \mathrm{~cm}, 1907$ (?) Document IFA, PT.DES.35/4. 


\section{Jean-Charles Moreux}

Lorsqu'il entre en architecture à l'École des beaux-arts de Paris en 1912, Jean-Charles Moreux dispose d'une certaine maturité et liberté de travail. II a une première formation d'ingénieur, ayant obtenu le diplôme de l'École spéciale des travaux publics de Paris en 1910. Moreux apprend à dessiner d'abord comme un ingénieur et devient architecte, avec l'attitude graphique du dessin technique. Afin d'approfondir sa culture classique en architecture, I'architecte organise, en 1926, après son parcours aux Beaux-Arts, un voyage d'étude en France et en Italie. D'Alberti à Claude Nicolas Ledoux, il retient en dessin tout ce qui l'intéresse et l'émeut de l'architecture urbaine et rurale classique. II étudie l'architecture des régions. Ses notes brèves et synthétiques lui apprennent à voir dans un jeu d'échelles multiples. Comme éventuel aide au projet, Moreux élabore des fiches thématiques restituant des aspects typologiques et morphologiques locaux. II installe souvent son dessin dans une relation au temps, comme une aide à la synthèse. II regarde le paysage dans la fenêtre mouvante d'un train et les points de vue s'enchaînent rapidement: ornement, édifice, ville, territoire. À partir de ses notes, Moreux comprend la dialectique formelle entre la structure et l'ornement, entre l'édifice et la rue, entre l'architecture et la nature. II apprend à voir la nature, sauvage et maîtrisée en même

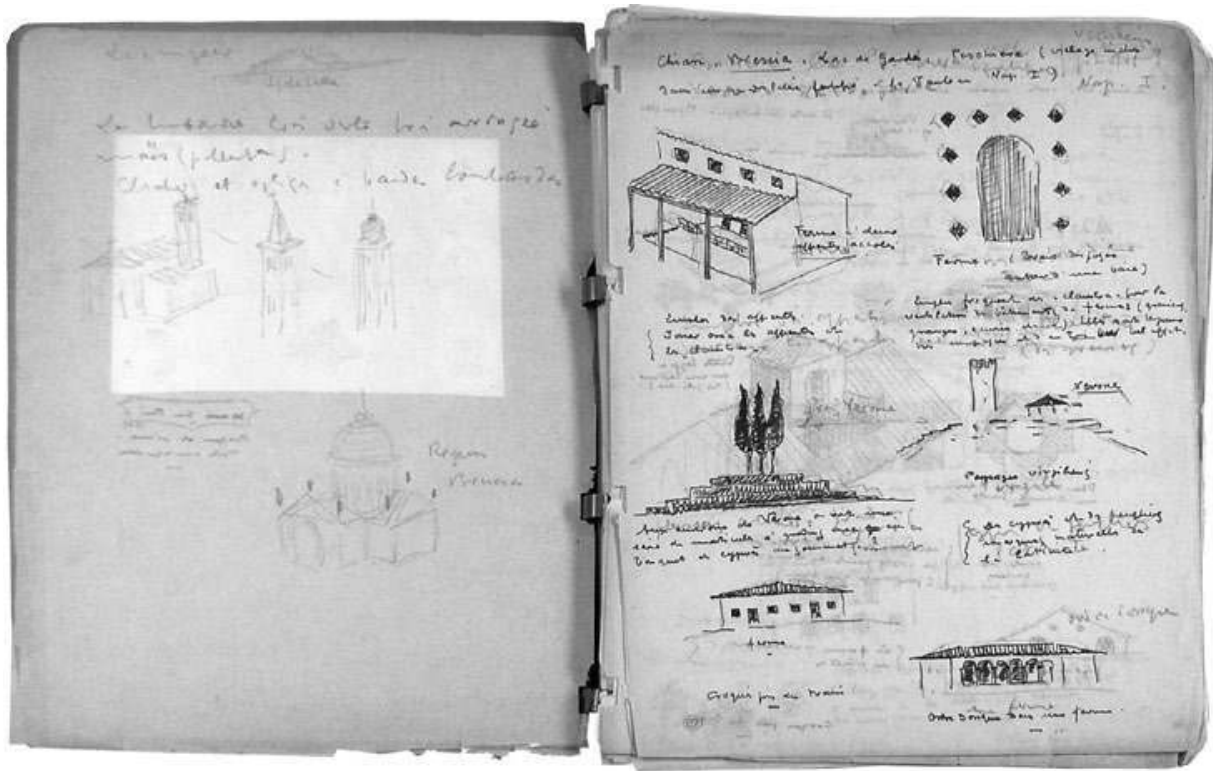

[fig. 2] Jean-Charles

Moreux, "Croquis

pris du train ", du

grand territoire au

détail grandeur, dessin

à la mine de plomb

puis à l'encre, carnet

$20 \times 25 \mathrm{~cm}, 1926$ (?)

Document IFA, 171.Ifa.4/1. 
temps, liée à l'architecture. Grâce au dessin de note, I'architecte forme sa pensée du grand territoire au détail grandeur. (Fig. 2)

\section{Charles-Édouard Jeanneret}

Nous avons également travaillé avec les dessins de note que Charles-Édouard Jeanneret trace lorsqu'il voyage pour son apprentissage au tournant du XIXe et du $x x^{e}$ siècle. Il reçoit en Suisse, à la Chaux-de-Fonds, une première formation d'art industriel et d'art décoratif, avant de devenir architecte, avant qu'il ne décide de devenir Le Corbusier à Paris en 1920. Jeanneret se forme très profondément au cœur du rapport art/industrie.

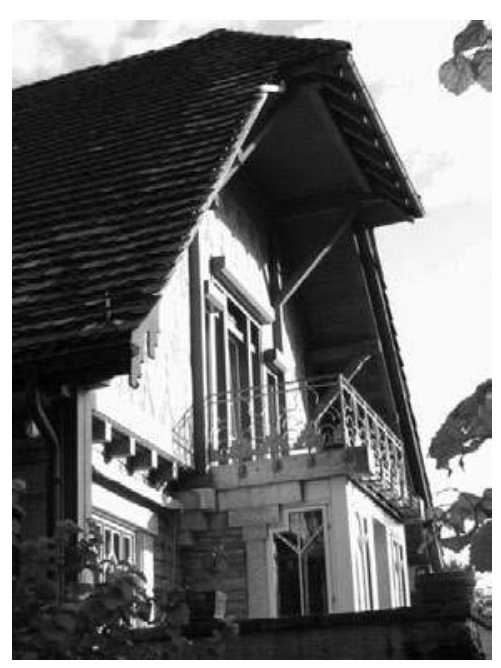

[fig. 3] Charles L'Eplattenier, René Chapallaz, Charles-Édouard Jeanneret et les élèves artisans du cours supérieur de l'école d'Art de la Chaux-deFonds, 1907. Ornement de la façade, Sgraffite, " style Sapin " par C.-É. Jeanneret. Photographie personnelle.
Grâce à son maître, le peintre neuchâtelois Charles L'Eplattenier, il est très tôt initié à l'art de la représentation et de l'abstraction, notamment à partir de l'invention de motifs et d'ornements issus des figures de la géométrie plane observées dans la nature locale: c'est le style Sapin. Jeanneret utilise la géométrisation pour comprendre et pour concevoir ${ }^{7}$. Après son cursus scolaire professionnel, il parcourt l'Europe afin d'acquérir une culture architecturale. II apprend à voir en voyage. "Si j'étais là-bas [c'est-à-dire à la Chaux-deFonds], comment voir par moi-même ${ }^{8}$ ? " Jeanneret regarde le monde localement, conscient des spécificités de chaque région, et le monde industriel. Lorsqu'il note, son dessin isole, extrait et décompose. II simplifie, radicalise et va à l'essentiel grâce à sa connaissance de la géométrie plane et des figures dans l'espace. (Fig. 3)

En 1910 Jeanneret est en Allemagne parce qu'il "étai [t] chargé d'une mission: étudier l'organisation de l'art décoratif en Allemagne" " pour la rédaction d'un ouvrage sur La Construction des villes ${ }^{10}$. Grâce à l'enseignement de L'Eplattenier et aux études urbaines de Camillo Sitte ${ }^{11}$, il analyse également la typologie des places urbaines des pays germaniques. Le jeune étudiant est particulièrement curieux de ces morphologies urbaines. Peut-être parce que la grille du plan de sa ville natale et horlogère reconstruite après I'incendie de 1794, ne lui avait pas donné le loisir de vivre dans un tissu urbain «médiéval ». Afin de retenir ce qui l'étonne et l'intéresse, l'étudiant annote des cartes postales illustrées. II semble que ce soit la première fois que Jeanneret exploite cet objet moderne et industrialisé pour recueillir ses impressions. Son intérêt pour la carte
7. Jean-Louis Cohen, Le Corbusier. La planète comme chantier, Paris, Textuel, 2005.

8. Charles-Édouard Jeanneret, "Paris, le 3 juillet 1908. Lettre à Monsieur L'Eplattenier ", dans Jean Petit, Le Corbusier lui-même, Genève, Rousseau, 1970, p. 31-34.
9. Le Corbusier, L'Art décoratif d'aujourd'hui, Paris, Crès, 1925, p. 209.

10. Étude commandée par Charles L'Eplattenier à la Chaux-de-Fonds à Charles-Édouard Jeanneret en 1909, organisant le « Voyage d'Allemagne » de 1910. Philippe Dubö̈, Architecture de la ville: culture et triomphe de l'urbanisme. Charles-Édouard Jeanneret, "La Construction des villes », Paris, MULT, 1985. Jean-Louis Cohen, op. cit. note 7.
11 Camillo Sitte, Des Städtebau nach seinen künstlerischen Grundsätzen, 1889. Édition consultée, Daniel Wieczorek pour la traduction française, L'Art de bâtir les villes, Paris, Éditions du Seuil, 1996. 
150 Les Cahiers de la recherche architecturale et urbaine - n³0/31

Trajectoires doctorales 2

[fig. 4] Charles-Édouard Jeanneret, carte postale illustrée annotée, retournement/détournement. "L'histoire des villes dans la ville ". Mine graphite et pastel sur carte postale $9 \times 14 \mathrm{~cm}$.

Document fonds « Le

Corbusier ", archives de

la bibliothèque de la ville de la Chaux-de-Fonds, LC/105/1112-13.

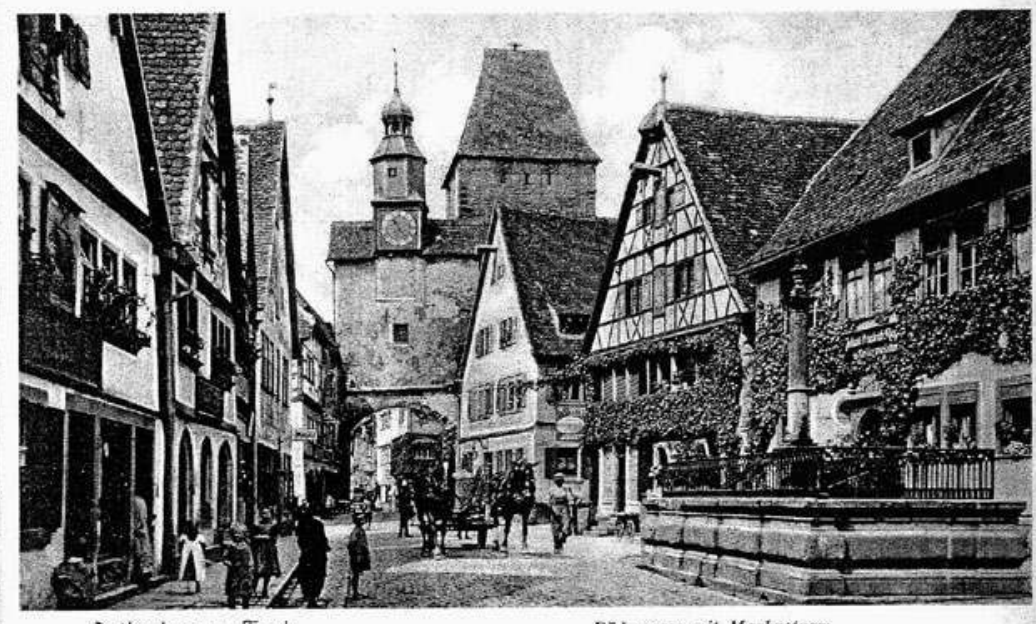

Rothenburg o. Caub.

Rödergasse mit Markusturm

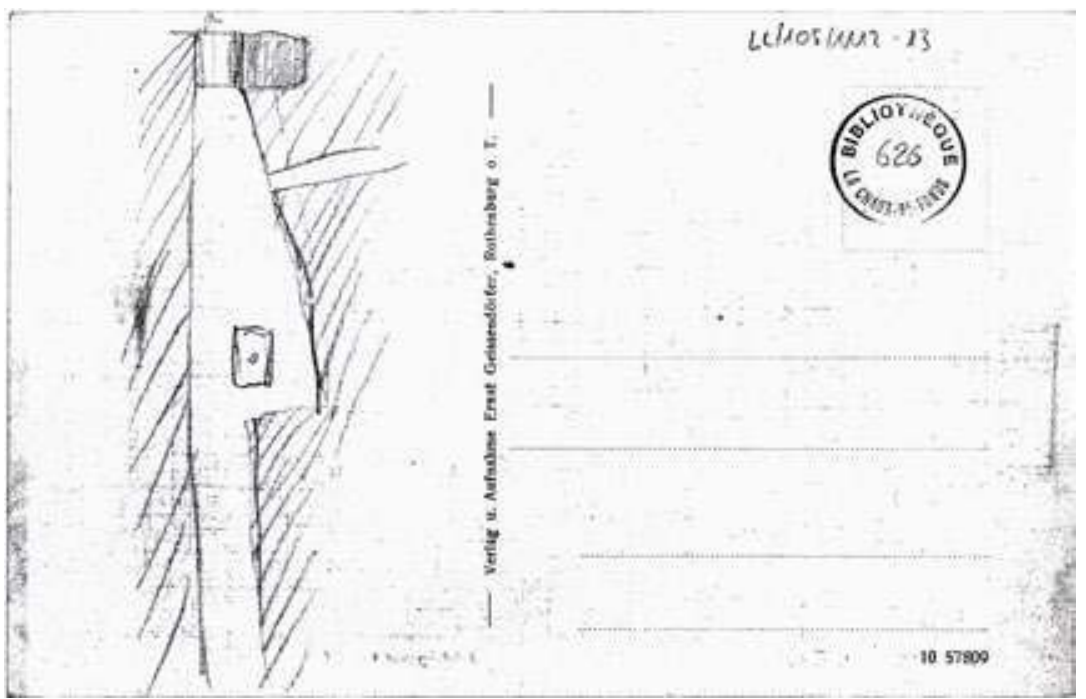


postale vient du système d'information qu'il met en place à partir du croisement entre carnets et photographies. En 1910, avec l'appareil photo Kodak $9 \times 9$ centimètres qu'il acquiert en 1907 pour sa vie d'étude en voyage et en "changement de terrain " ${ }^{12}$, Jeanneret s'initie encore au travail visuel de la photographie. L'utilisation de sa "caméra » n'est pas systématique. Ce n'est pas encore l'outil de la note et du spontané, comme peut l'être la carte postale. (Fig. 4)

L'aire visuelle de cet instrument fonctionne en double face et retournement cadrés. La page illustrée montre une représentation neutre de l'existant. Par interdessin, I'architecte en transcrit sa perception. II exploite l'image, la représentation déjà là. II se concentre sur ce qu'il voit. Il redessine et progressivement passe au schéma, l'image devient intellection. II y a un dessin sous le dessin et dans le dessin, le support devient subjectile.

Dans La Carte postale. De Socrate à Freud et au-delà, Jacques Derrida nous engage: "Regarde de l'autre côté. » La carte postale est réversible. Jeanneret annote souvent « Voir derrière ». En un tour de carte, le subjectile présente une page blanche. La page blanche est une abstraction ouverte à toute histoire. L'architecte y relève son choix. Avec la carte postale et l'image, déjà, Jeanneret peut penser au dessin avant le dessin et le construire selon sa perception. II pense au trait avant la ligne. Le trait ${ }^{13}$ rend visible une idée d'une matérialité constructive. Il est d'ordre conceptuel. Afin d'approfondir son regard, Jeanneret assemble différents systèmes de représentation. Et dans un jeu déjà dadaïste, il travaille en ready made, si l'objet industrialisé est retransformé en unicum par le dessin. (Fig. 5)
Le dessin de note devient pour Jeanneret un mode d'apprentissage. II emprunte ses acquis graphiques, théoriques et pratiques, en arts visuels, industriels et décoratifs, pour construire sa culture classique en architecture et fonder un regard moderne sur le monde édifié et le monde à venir. Jeanneret s'intéresse au lien entre matérialité (innovation technologique) et philosophie visuelle (la représentation comme théorie de la connaissance et spécificité de pensée). Jeanneret utilise le dessin de note pour comprendre la grande culture de l'architecture et connaître les nécessités du métier. II utilise sa culture géométrique initiale pour apprendre à voir en trois dimensions. L'alliance des figures de la géométrie plane et les figures de l'espace est un système efficace de compréhension de l'architecture et d'expression du regard de l'architecte. La géométrie devient une véritable méthode de synthèse et force peut-être au "schème transcendantal" kantien, cette entité qui participe du monde sensible des émotions au monde intelligible des idées et qui permet de transcender une intuition en concept pur de l'entendement.

\section{La géométrie est un facteur du visible}

La géométrie organise un dessin intellectuel et sensible entre ratio et phaenomena. Le dessin géométrique fabrique une clarification d'une réalité complexe. II retrace les différentes couches conceptuelles du monde édifié, dans un langage scientifique et visuel, à visée universelle. C'est une réduction vers l'essentiel qui participe de la transformation de la forme (Idea), du lineamenta ${ }^{14}$ qu'Alberti distingue comme dessin en soi
12. Louis Althusser, op. cit. note 3.

13 Hubert Damisch, Le Traité du trait, Paris, Réunion des musées nationaux, 1995.
14. Leon Battista Alberti, De rRe aAedificatoria, 1485. Édition consultée, établie par Françoise Choay et Pierre Caye pour la traduction française du latin, Paris, Le Seuil, 2004, p. 56. 


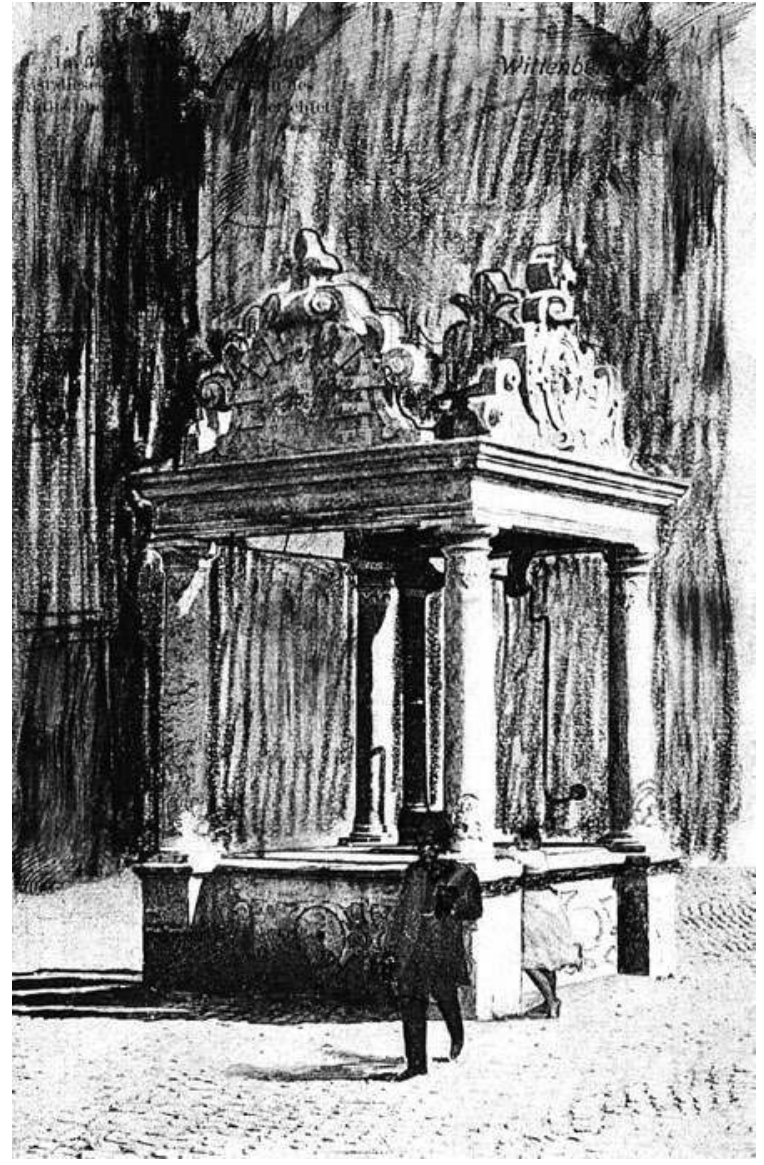

[fig. 5] Charles-Édouard

Jeanneret, carte postale

illustrée annotée, retournement/

détournement. "L'architec-

ture classique et le monde

contemporain ". Mine graphite

et pastel sur carte postale

$9 \times 14 \mathrm{~cm}$.

Document fonds « Le Corbusier 》,

archives de la bibliothèque

de la ville de la Chaux-de-Fonds,

LC/105/1112-26.

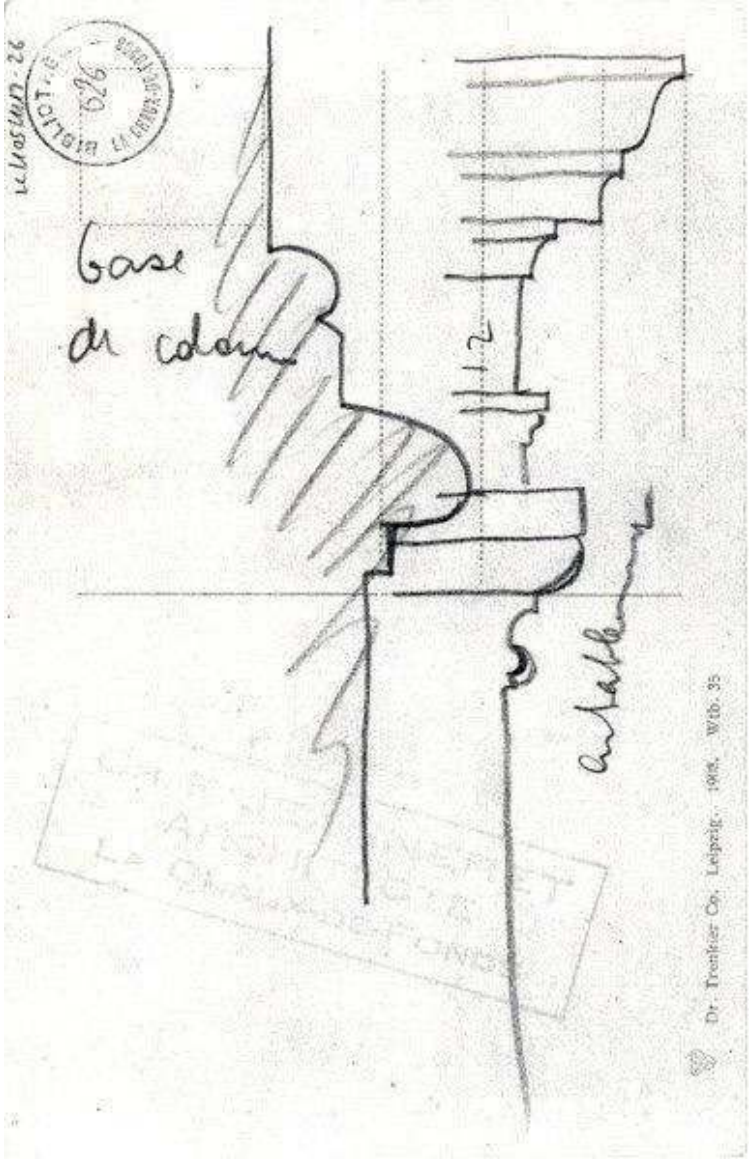


qui ne dépend pas de la matière, en lineamentum, en dessin de l'architecture physique pour la mise en œuvre et l'organisation de la construction ("Prescriptio »). En tant que processus d'abstraction et de figuration sensible, la géométrie est un facteur du visible. Elle offre à l'architecte le moyen de comprendre et de mesurer le monde anthropologique puis de l'organiser en monde bâti, puisqu'elle engage la topographie et la chorographie. L'architecte des Team X, Giancarlo De Carlo tente ainsi le territoire et passe du projet à la tentative. L'architecte réhabilite le bourg médiéval de Colletta di Castelbianco, fondé en dénivelé sur les pentes d'une colline du Piémont. II appréhende le site et ses reliefs transversaux en le décomposant grâce à la géométrie. Le village est composé de cellules définies par la pente et reliées les unes aux autres comme le ventricule d'un crustacé - autonomes et collectives en même temps. Giancarlo De Carlo exploite la géométrie du sol pour tenter le territoire, liée à celle du projet. II propose plusieurs solutions et combinaisons d'articulations pour restaurer et actualiser les cellules. De concert, il laisse au lieu son histoire et les spécificités de sa conception initiale. La géométrie permet le passage de l'analyse au projet, de l'idée à la discussion constructive, en reliant la main, l'œil et I'esprit ${ }^{15}$. Ce procédé graphique appliqué à l'architecture construit une représentation d'objet existant ou ayant existé, à fabriquer ou imaginaire. Il est à acquérir précisément pour aller vite et juste dans son dessin, et le développement de sa maîtrise technique est sans cesse pertinent dans l'enseignement dispensé au sein des écoles d'architecture.

\section{La pensée par le visuel à l'heure du dessin numérique}

D'autant, I'usage aujourd'hui des outils numériques dans la nécessité du métier et de la formation de l'architecte, mène à reprendre cette question de la pensée par le visuel, pour l'interprétation du réel et la composition du monde physique. Le jeune architecte doit d'abord comprendre comment la machine fonctionne et trace à l'écran, afin qu'elle devienne un véritable outil d'analyse et de conception. Le dessin numérique est fondé sur la géométrie spatiale ${ }^{16}$ qui permet les allers-retours 2D/3D, forme/matériau, calcul/tracé, figures planes/ spatialité, architecture de l'arithmétique/architecture de la règle et du compas: scibilité ${ }^{17}$. Le trait projeté est une ligne algébrique et rationnelle qui détient une mesure. L'association géométrie/dessin numérique semble être un système visuel par lequel l'architecte peut exercer son œil à voir. Comment les outils numériques participent-ils de l'acquisition de la culture visuelle de l'architecte et de sa transformation en regard instruit?

Nous souhaitons également explorer les qualités de temps que l'ordinateur propose à l'architecte qui dessine pour apprendre à apprendre. Le temps que l'architecte accorde à son dessin, la vitesse du trait, proposent un statut. La position du dessinateur et la posture de son œil peuvent intervenir également. Le temps bref de la note semble forcer à la synthèse. Qu'en est-il de la rythmique du dessin numérique, des temps courts et des temps longs à partir de la souris? Est-ce la relation à la vitesse qui permet d'aller à l'essentiel, vers une forme courte de représentation? Ou bien est-ce la mesure du trait, le dessin construit et adapté à la
15. Maurice Merleau-Ponty, L'Eil et l'Esprit, Paris, Gallimard, 1964.
16. Daniel Jacques, Jean-François Calame, Géométrie spatiale. Le vade-mecum, Lausanne, PPUR, 2013.

17. Athanase Papadopoulos, «Degrés de complexité en géométrie et en musique. Réflexions à partir de L'Harmonie du monde de Kepler », Strasbourg, Irma, université Louis-Pasteur et CNRS, 2007. 
perception? Le temps retenu par la réflexion graphique pour la représentation et la composition, suggère-t-il un dessin plus efficace à la construction du regard de l'architecte? L'ordinateur admet-il différents tempi du dessin et d'accroche du trait, adaptables à la perception visuelle? "L'œil est la chose la plus puissante, la plus rapide et la plus précieuse » " nous dit déjà Alberti. L'organe savant de l'architecte trouve le dessin comme modalité de restitution de sa perception. C'est pourquoi nous proposons d'approfondir la question de la transformation de la culture visuelle de l'architecte en pensée architecturale, et de ses systèmes de mise en œuvre, grâce au contexte des Visual Studies 